\title{
On unbounded commuting Jacobi operators and some related issues
}

https://doi.org/10.1515/conop-2019-0008

Received May 22, 2018; accepted August 5, 2019

Abstract: We consider the situations, when two unbounded operators generated by infinite Jacobi matrices, are self-adjoint and commute. It is found that if two Jacobi matrices formally commute, then two corresponding operators are either self-adjoint and commute, or admit a commuting self-adjoint extensions. In the latter case such extensions are explicitly described. Also, some necessary and sufficient conditions for self-adjointness of Jacobi operators are studied.

Keywords: Jacobi matrices, Self-adjoint extensions, Moment problem, Orthogonal polynomials.

MSC: 47B39, 47A10, 39A70

\section{Introduction}

In operator theory, the study of commuting operators remains an important topic [2]. We recall that two self-adjoint (possibly unbounded) operators (or, more generally, two normal operators) are said to commute if their spectral projections commute. In [1] E. Nelson showed an example of two essentially self-adjoint operators $A$ and $B$ in a Hilbert space $\mathcal{H}$ having a common dense domain $\mathcal{D}$ such that for all $x$ in $\mathcal{D}, A B x=$ $B A x$, but such that $\bar{A}$ and $\bar{B}$ do not commute (where $\bar{A}$ stands for the closure of $A$ ). He proved the following sufficient conditions for the commutativity of two operators:

Theorem, Nelson. Let $A$ and $B$ be symmetric operators in a Hilbert space $\mathcal{H}$ and let $\mathcal{D}$ be a dense linear manifold in $\mathcal{H}$ such that $\mathcal{D}$ is contained in the domain of $A^{2}, B^{2}, A B$ and $B A$ and such that $A B x=B A x$ for all $x \in \mathcal{D}$. If the restriction of $A^{2}+B^{2}$ on $\mathcal{D}$ is essentially self-adjoint then $A$ and $B$ are essentially self-adjoint and $\bar{A}$ and $\bar{B}$ commute.

Another proof of Nelson's theorem (which does not use operator representations of Lie algebras) was offered in the paper of A.E. Nussbaum [3], which contains some results on commutativity of two operators. In case of the Nelson's example, it was shown in [3] (Theorem 5) that for the commutativity of $\bar{A}$ and $\bar{B}$ some additional conditions are required:

Theorem, Nussbaum. Let $A$ and $B$ be symmetric operators in a Hilbert space $\mathcal{H}$ and let $\mathcal{D}$ be a dense linear manifold in $\mathcal{H}$ such that $\mathcal{D}$ is contained in the domain of $A$ and $B$, and such that $(A x, B y)=(B x, A y)$ for all $x$ and $y$ in $\mathcal{D}$. Let $T$ be the closure of the restriction of $A+B$ to $\mathcal{D}$. If $T$ is self-adjoint and its domain $D(T) \subset D(\bar{A})$. then $A$ and $B$ are essentially self-adjoint and $\bar{A}$ and $\bar{B}$ commute.

In this paper, we mainly study the commutativity of Jacobi operators, i. e. the operators generated by infinite Jacobi matrices. Note that the commutativity of a certain class of block Jacobi operators (i. e. Jacobi operators with matrix elements) is important in the theory of orthogonal polynomials of several variables and multidi-

^Corresponding Author: Andrey Osipov: Federal State Institution "Scientific-Research Institute for System Analysis of the Russian Academy of Sciences", Nakhimovskii pr. 36-1, Moscow, 117218. E-mail: osipa68@yahoo.com 
mensional moment problem ( see [4, 8, 9]), and there are some open questions there (e. g. the commutativity of block Jacobi operators, which satisfy the Carleman type condition). We believe that our results may be useful for studying the commutativity of such operators and plan to address these issues in a further paper.

The paper is organized as follows. In the section to follow, we consider the general case of Jacobi operators and study the conditions for their self-adjointness, including some criteria. In Section 3, we consider the nonself-adjoint Jacobi operators and offer a procedure for their self-adjoint extension. In the final section, we study the commutativity of a couple of Jacobi operators generated by commuting Jacobi matrices.

\section{Some results about the self-adjointness of Jacobi operators.}

First, we recall some facts from the theory of Jacobi operators, necessary for our further considerations [5$7,12]$. An infinite Jacobi matrix is defined to be matrix of the form:

$$
J=\left(\begin{array}{cccc}
b_{0} & a_{0} & 0 & 0 \\
a_{0} & b_{1} & a_{1} & 0 \\
0 & \ddots & \ddots & \ddots
\end{array}\right)
$$

where $a_{n}, b_{n} \in \mathbb{R}, a_{n}>0, n \in \mathbb{Z}_{+}$.

To the matrix $J$ we assign the second-order difference equation:

$$
l(y)_{n}:=a_{n-1} y_{n-1}+b_{n} y_{n}+a_{n} y_{n+1}=\lambda y_{n}, \lambda \in \mathbb{C}, a_{-1}=1 .
$$

It has two linearly independent solutions $\left(P_{i}(\lambda)\right)_{i=-1}^{\infty}$ and $\left(Q_{i}(\lambda)\right)_{i=-1}^{\infty}$ with the initial conditions

$$
P_{-1}(\lambda)=0, P_{0}(\lambda)=1, \quad Q_{-1}(\lambda)=-1, Q_{0}(\lambda)=0 ;
$$

so any solution of (1) is a linear combination of them. The function $P_{i}(\lambda)$ is a polynomial of degree $i$ in $\lambda$ and is called a polynomial of the first kind, while $Q_{i}(\lambda)$ is a polynomial of degree $i-1$ and is called a polynomial of the second kind. Note that $P(\lambda):=\left(P_{i}(\lambda)\right)_{i=0}^{\infty}$ is a solution of the equation $J y=\lambda y$, but $Q(\lambda):=\left(Q_{i}(\lambda)\right)_{i=0}^{\infty}$ is not: $(J Q)_{0}=1 \neq 0=\lambda Q_{0}$.

The analogue of Liouville's formula holds for $P(\lambda)$ and $Q(\lambda)$ :

$$
P_{i}(\lambda) Q_{i+1}(\lambda)-P_{i+1}(\lambda) Q_{i}(\lambda)=\frac{1}{a_{i}}, \quad i \in \mathbb{Z}_{+} .
$$

The Wronskian of two solutions $y=\left(y_{n}(\lambda)\right)_{n=-1}^{\infty} z=\left(z_{n}(\lambda)\right)_{n=-1}^{\infty}$ of (1) is defined to be

$$
W_{n}(y, z):=a_{n}\left(y_{n} z_{n+1}-y_{n+1} z_{n}\right) .
$$

It follows from (2) that $W_{n}(y, z)$ does not depend on $n$; two solutions of (1) are linearly independent if and only if their Wronskian is nonzero.

Let $y=y(\lambda)$ be a solution of (1) with the parameter $\lambda$, and $z=z(\mu)$ a solution of the same equation with the parameter $\mu$. Then it is easy to verify the Green's formula:

$$
\sum_{i=m}^{N} y_{i} l(z)_{i}-l(y)_{i} z_{i}=(\mu-\lambda) \sum_{i=m}^{N} y_{i} z_{i}=a_{N}\left(y_{N} z_{N+1}-y_{N+1} z_{N}\right)-a_{m-1}\left(y_{m-1} z_{m}-y_{m} z_{m-1}\right),
$$

where $m$ and $N$ are arbitrary nonnegative integers.

Our next aim is to pass from the matrix $J$ to Jacobi operators. For this purpose we introduce the Hilbert space $l^{2}[0, \infty)$ consisting of the complex sequences $y=\left(y_{n}\right)_{n=0}^{\infty}$ such that $\sum_{n=0}^{\infty}\left|y_{n}\right|^{2}<\infty$, with the inner product $(y, z)=\sum_{n=0}^{\infty} y_{n} \bar{z}_{n}$. Denote by $\left(e_{n}\right)_{n=0}^{\infty}$ its standard orthonormal basis. Then we introduce the linear set $D$ of all elements $y \in l^{2}[0, \infty)$ such that $J y \in l^{2}[0, \infty)$. We define the operator $L$ on $D$ by the equality $L y=J y$. For $y$ and $z$ from $D$, similarly to (3) we have

$$
\sum_{n=0}^{N} y_{n}(J \bar{z})_{n}-(J y)_{n} \bar{z}_{n}=[y, z]_{N}
$$


where $[y, z]_{N}:=a_{N}\left(y_{N} \bar{z}_{N+1}-y_{N+1} \bar{z}_{N}\right)$. Since $J y, J \bar{z}$ are in $l^{2}[0, \infty)$, we find that the limit $[y, z]_{\infty}:=$ $\lim _{N \rightarrow \infty}[y, z]_{N}$ exists and is finite, so we get

$$
(y, L z)-(L y, z)=[y, z]_{\infty} .
$$

Consider the dense linear set $D_{0}^{\prime} \in l^{2}[0, \infty)$ consisting of finitely nonzero vectors. Denote by $L_{0}^{\prime}$ the restriction of the operator $L$ to $D_{0}^{\prime}$. As follows from (4), $L_{0}^{\prime}$ is symmetric $\left([y, z]_{\infty}=0\right)$. Consequently, it admits closure. Denote its closure by $L_{0}$. The domain $D_{0}$ of $L_{0}$ consists of precisely those vectors $y \in D$ which satisfy the condition

$$
[y, z]_{\infty}=0, \quad \forall z \in D .
$$

The operator $L_{0}$ is a closed symmetric operator with deficiency indices $(0,0)$ or $(1,1)$ (it is said that the limitpoint or limit-circle case holds for $L_{0}$ ), and the operator $L$ is the adjoint of $L_{0}$. Thus, if the limit-point case holds, then $L_{0}=L$. In the limit-circle case the linear space of solutions $J y=\lambda y$ from $l^{2}[0, \infty)$ is at most onedimensional: $y=y_{0} P(\lambda)$ ). The operators $L_{0}$ and $L$ are called, respectively, the minimal and maximal (Jacobi) operators generated by the matrix $J$.

For the operator $L_{0}$, the function $\rho(\lambda)=\left(E_{\lambda} e_{0}, e_{0}\right)$, where $E_{\lambda}$ is a (generalized) spectral function of $L_{0}$ (more details and definitions can be found in $[5,6,17]$ ) yields a solution of the Hamburger moment problem, and we have

$$
s_{n}:=\left(J^{n}\right)_{0,0}=\left(L_{0}^{n} e_{0}, e_{0}\right)=\int_{-\infty}^{\infty} \lambda^{n} d \rho(\lambda) \quad n \in \mathbb{Z}_{+} .
$$

Thus, we can talk about the Hamburger moment problem associated with $L_{0}$ (or with $J$ ). The polynomials $P_{i}(\lambda)$ are orthogonal with respect to $d \rho(\lambda)$ :

$$
\int_{-\infty}^{\infty} P_{i}(\lambda) P_{j}(\lambda) d \rho(\lambda)=\delta_{i, j}, \quad i, j \in \mathbb{Z}_{+} ;
$$

and for $Q_{i}(\lambda)$ the following representation holds

$$
Q_{i}(\lambda)=\int_{-\infty}^{\infty} \frac{P_{i}(\lambda)-P_{i}(\mu)}{\lambda-\mu} d \rho(\mu) .
$$

From the orthogonality of $P(\lambda)$ and (1) follows that

$$
a_{n}=\int_{-\infty}^{\infty} \lambda P_{n}(\lambda) P_{n+1}(\lambda) d \rho(\lambda), \quad b_{n}=\int_{-\infty}^{\infty} \lambda P_{n}(\lambda) P_{n}(\lambda) d \rho(\lambda), \quad n \in \mathbb{Z}_{+} .
$$

The above formulae give the reconstruction procedure for the matrix $J$ (operator $L_{0}$ ) from $d \rho(\lambda)$ : namely, first, applying the Gram-Schmidt orthogonalization process to the sequence $\left(\lambda^{i}\right)_{i=0}^{\infty}$, we get $P(\lambda)$. Then we find the elements of $J$ by (7). Note that $a_{n}$ and $b_{n}$ are the two leading Fourier coefficients (we call them principal ones) of the functions $\lambda P_{n}(\lambda)$ with respect to the system $P(\lambda)$.

If the deficiency indices of $L_{0}$ are maximal ( $\left.(1,1)\right)$, it means that for the Hamburger moment problem associated with $L_{0}$ the indeterminate case holds (i. e. it has multiple solutions, see [6] for details). In this case both $P(\lambda)$ and $Q(\lambda)$ belong to $l^{2}[0, \infty)$ for all complex values of $\lambda$. Conversely, if $P(\lambda)$ and $Q(\lambda)$ belong to $l^{2}[0, \infty)$ for all $\lambda \in \mathbb{C}$, then for all non-real $\lambda$ the solution of equation $J P(\lambda)=\lambda P(\lambda)$ belongs to this space, therefore the deficiency subspaces of $L_{0}$ are one-dimensional, which means that the deficiency indices of $L_{0}$ are $(1,1)$. This condition is fulfilled if at least for one $\lambda=\lambda_{0} \in \mathbb{C}$, both $P\left(\lambda_{0}\right)$ and $Q\left(\lambda_{0}\right)$ are from $l^{2}[0, \infty)$ (for the operators generated by Jacobi block matrices (block Jacobi operators) the proof of a similar fact (that the deficiency indices of the block counterpart of $L_{0}$ are maximal) is contained in [16], Theorem 2). To prove this " $l$ - property" for $P(\lambda)$, we first check that

$$
\frac{P_{i}(\lambda)-P_{i}\left(\lambda_{0}\right)}{\lambda-\lambda_{0}}=\sum_{j=0}^{i-1} K_{i, j} P_{j}(\lambda), \quad i \in \mathbb{N}
$$


(indeed, the right hand side of this equation is a polynomial of degree $i-1$, and therefore is expandable into a linear combination of $P_{0}(\lambda), \ldots, P_{i-1}(\lambda)$ ). The coefficients $K_{i, j}$ are determined by the Fourier formulae [13]:

$$
\begin{aligned}
K_{i, j} & =K_{i, j}\left(\lambda_{0}\right)=\int_{-\infty}^{\infty} \frac{P_{i}(\lambda)-P_{i}\left(\lambda_{0}\right)}{\lambda-\lambda_{0}} P_{j}(\lambda) d \rho(\lambda)= \\
& =P_{j}\left(\lambda_{0}\right) \int_{-\infty}^{\infty} \frac{P_{i}(\lambda)-P_{i}\left(\lambda_{0}\right)}{\lambda-\lambda_{0}} d \rho(\lambda)+\int_{-\infty}^{\infty}\left(P_{i}(\lambda)-P_{i}\left(\lambda_{0}\right)\right) \frac{P_{j}(\lambda)-P_{j}\left(\lambda_{0}\right)}{\lambda-\lambda_{0}} d \rho(\lambda)= \\
& =P_{j}\left(\lambda_{0}\right) Q_{i}\left(\lambda_{0}\right)-P_{i}\left(\lambda_{0}\right) Q_{j}\left(\lambda_{0}\right)=\left|\begin{array}{ll}
P_{j}\left(\lambda_{0}\right) & Q_{j}\left(\lambda_{0}\right) \\
P_{i}\left(\lambda_{0}\right) & Q_{i}\left(\lambda_{0}\right)
\end{array}\right|, \quad j=0, \ldots, i-1 ;
\end{aligned}
$$

here we used the representation (6) for $Q(\lambda)$ and the fact that $P_{i}(\lambda)$ is orthogonal to polynomials of lower degree. Thus we get

$$
P_{i}(\lambda)=P_{i}\left(\lambda_{0}\right)+\left(\lambda-\lambda_{0}\right) \sum_{j=0}^{i-1} K_{i, j} P_{j}(\lambda) .
$$

Substituting (9) into (6), we find a similar representation for $Q_{i}(\lambda)$ :

$$
Q_{i}(\lambda)=Q_{i}\left(\lambda_{0}\right)+\left(\lambda-\lambda_{0}\right) \sum_{j=0}^{i-1} K_{i, j} Q_{j}(\lambda) .
$$

The claimed property for $P(\lambda)$ and $Q(\lambda)$ then follows from Lemma 1.3.2 of [6]. In view of the above considerations, we obtain the following criterion of self-adjointness for $L_{0}$.

Theorem 1. The operator $L_{0}$ is self-adjoint iff there exists $\lambda_{0} \in \mathbb{C}$ such that

$$
\sum_{i=0}^{\infty}\left|P_{i}\left(\lambda_{0}\right)\right|^{2}+\left|Q_{i}\left(\lambda_{0}\right)\right|^{2}=\infty .
$$

If (10) is fulfilled, then

$$
\sum_{i=0}^{\infty}\left|P_{i}(\lambda)\right|^{2}+\left|Q_{i}(\lambda)\right|^{2}=\infty \quad \text { for all } \lambda \in \mathbb{C} .
$$

As we see, the coefficients $K_{i, j}$ depend on $\lambda_{0}$. In what follows, unless the other is specified, we will set $\lambda_{0}=0$.

For the (minimal) operators generated by block Jacobi matrices, the criterion of maximality for their deficiency indices in terms of $K_{i, j}$ (which were defined similarly to (8)) was established in [14,15]. Utilizing this result for the scalar Jacobi operators $L_{0}$ ( whose deficiency indices are at most $(1,1)$ ), we get another criterion of self-adjointness.

Theorem 2. The operator $L_{0}$ is self-adjoint iff there exists a sequence of intervals of positive integers $\left[n_{k}, m_{k}\right]$ such that $m_{k} \leq n_{k+1}<m_{k+1},(k \in \mathbb{N})$ for which the following condition is satisfied:

$$
\sum_{k=1}^{\infty}\left(\sum_{i=n_{k}}^{m_{k}} \sum_{j=n_{k}}^{i}\left|K_{i, j}\right|^{2}\right)^{1 / 2}=\infty .
$$

Proof. Assume $L_{0}$ is self-adjoint. According to the previous theorem, this implies that

$$
\sum_{i=0}^{\infty}\left|P_{i}\right|^{2}+\left|Q_{i}\right|^{2}:=\sum_{i=0}^{\infty}\left|P_{i}(0)\right|^{2}+\left|Q_{i}(0)\right|^{2}=\infty .
$$

As follows from (8)

$$
\left|K_{i, j}\right|^{2} \leq\left(\left|P_{j}\right|\left|Q_{i}\right|+\left|P_{i}\right|\left|Q_{j}\right|\right)^{2} \leq\left(\left|P_{i}\right|^{2}+\left|Q_{i}\right|^{2}\right)\left(\left|P_{j}\right|^{2}+\left|Q_{j}\right|^{2}\right) .
$$


Take an arbitrary sequence $\left[n_{k}, m_{k}\right]$. We have

$$
\sum_{i=n_{k}}^{m_{k}} \sum_{j=n_{k}}^{i}\left|K_{i, j}\right|^{2} \leq \sum_{i=n_{k}}^{m_{k}}\left(\left|P_{i}\right|^{2}+\left|Q_{i}\right|^{2}\right) \sum_{j=n_{k}}^{i}\left(\left|P_{j}\right|^{2}+\left|Q_{j}\right|^{2}\right) \leq\left(\sum_{i=n_{k}}^{m_{k}}\left|P_{i}\right|^{2}+\left|Q_{i}\right|^{2}\right)^{2} .
$$

Taking the square root from both sides of the obtained inequality and summing over $k$, we get

$$
\sum_{k=1}^{\infty}\left(\sum_{i=n_{k}}^{m_{k}} \sum_{j=n_{k}}^{i}\left|K_{i, j}\right|^{2}\right)^{1 / 2} \leq \sum_{k=1}^{\infty}\left(\sum_{i=n_{k}}^{m_{k}}\left|P_{i}\right|^{2}+\left|Q_{i}\right|^{2}\right) \leq 2 \sum_{i=0}^{\infty}\left(\left|P_{i}\right|^{2}+\left|Q_{i}\right|^{2}\right) .
$$

Hence, as follows from (12), the condition (11) is fulfilled for $\left[n_{k}, m_{k}\right]$. Thus the necessity is proved. Now assume that (11) is fulfilled for a certain sequence $\left[n_{k}, m_{k}\right]$. As follows from (13), the condition (12) is fulfilled as well. By the previous theorem, the latter implies that $L_{0}$ is self-adjoint.

This criterion leads to the following important result about self-adjointness of $L_{0}$.

Corollary. If for an arbitrary fixed $p \in \mathbb{N}$

$$
\sum_{n=0}^{\infty}\left|K_{n+p, p}\right|=\infty
$$

then the operator $L_{0}$ is self-adjoint.

Proof. Consider the set of $p$ sequences: $[s+n p, s+(n+1) p]_{n=0}^{\infty}, s=1, \ldots, p$. One can check that for any $n$ the element $\left|K_{n+p, p}\right|^{2}$ is contained in the sum

$$
\sum_{i=s+n p}^{s+(n+1) p} \sum_{j=s+n p}^{i}\left|K_{n+p, p}\right|^{2}
$$

for some $s$. Hence, we get

$$
\sum_{s=1}^{p} \sum_{n=0}^{\infty}\left(\sum_{i=s+n p}^{s+(n+1) p} \sum_{j=s+n p}^{i}\left|K_{n+p, p}\right|^{2}\right)^{1 / 2} \geq \sum_{n=1}^{\infty}\left|K_{n+p, p}\right|
$$

(for $p=1$, we have an equality here). If the series in the right side of this inequality is divergent, then at least one of $p$ series in the left side is divergent too; therefore $L_{0}$ is self-adjoint.

Thus, for different values of $p$, we have a number of sufficient conditions for the operator $L_{0}$ to be self-adjoint. As noted in [14], some of these conditions coincide with the previously known results on the Jacobi operators (see also [11], where various sufficient conditions are studied). For example, for $p=1$ we find using (2) that

$$
K_{n+1, n}=P_{n} Q_{n+1}-P_{n+1} Q_{n}=\frac{1}{a_{n}},
$$

so the condition (14) in this case becomes

$$
\sum_{n=0}^{\infty} \frac{1}{a_{n}}=\infty
$$

which is the well-known Carleman test (see e. g. $[6,12]$ for details). For $p=2$ we have

$$
K_{n+2, n}=\left|\begin{array}{cc}
P_{n} & Q_{n} \\
P_{n+2} & Q_{n+2}
\end{array}\right|=\left|\begin{array}{cc}
P_{n} & Q_{n} \\
-\frac{b_{n+1}}{a_{n+1}} P_{n+1} & -\frac{b_{n+1}}{a_{n+1}} Q_{n+1}
\end{array}\right|=-\frac{b_{n+1}}{a_{n} a_{n+1}} .
$$

In this case, (14) coincides with the Dennis-Wall test, [6]:

$$
\sum_{n=0}^{\infty} \frac{\left|b_{n+1}\right|}{a_{n+1} a_{n}}=\infty
$$


Acting similarly, we obtain the recurrent relations for $p>2$

$$
K_{n+p, n}=\left|\begin{array}{cc}
P_{n} & Q_{n} \\
P_{n+p} & Q_{n+p}
\end{array}\right|=-\frac{b_{n+p-1}}{a_{n+p-1}} K_{n+p-1, n}-\frac{a_{n+p-2}}{a_{n+p-1}} K_{n+p-2, n} .
$$

Also, from (15)-(17) we find

$$
a_{n}=\frac{1}{K_{n+1, n}}, \quad b_{n+1}=-\frac{K_{n+2, n}}{K_{n+1, n} K_{n+2, n+1}} ; \quad n \in \mathbb{Z}_{+} .
$$

Thus, all elements of the martix $J$ except $b_{0}$, as well as $K_{n+p, n}$ for $p>2$ can be expressed through $K_{n+1, n}, K_{n+2, n}, n \in \mathbb{Z}_{+}$. If we define $K_{1,-1}$ similarly to (8)

$$
K_{1,-1}:=\left|\begin{array}{cc}
P_{-1} & Q_{-1} \\
P_{1} & Q_{1}
\end{array}\right|,
$$

we get the formula for $b_{0}$

$$
b_{0}=-\frac{K_{1,-1}}{K_{1,0}} .
$$

As follows from (8), $K_{n, n-1}, K_{n, n-2}$ are the principal Fourier coefficients of the functions $\tilde{P}_{n}(\lambda):=\frac{P_{n}(\lambda)-P_{n}(0)}{\lambda}$ with respect to the system $P(\lambda)$. Set $\tilde{P}(\lambda)=\left(\tilde{P}_{n}(\lambda)\right)_{n=1}^{\infty}$. Now we can make the following definition.

Definition. We call the set $\operatorname{PFC}(J, \tilde{P}(\lambda)):=\left(\left\{K_{n, n-2}\right\},\left\{K_{n, n-1}\right\}\right)_{n=1}^{\infty}$ with the elements, defined according to (8),(20), the principal Fourier coefficients of the functions $\tilde{P}(\lambda)$ with respect to the system $P(\lambda)$, corresponding to the matrix $J$.

As follows from the above calculations, the set $\operatorname{PFC}(J, \tilde{P}(\lambda))$ is equivalent to the set of elements of the matrix $J:\left(b_{n}, a_{n}\right)_{n=0}^{\infty}$, which, as noted above, are in turn the principal Fourier coefficients of the functions $\lambda P(\lambda)$ with respect to the system $P(\lambda)$. Therefore, we may associate the operator $L_{0}$ with $P F C(J, \tilde{P}(\lambda))$.

Now consider the matrix $J$ with zero main diagonal: $b_{n}=0, n \in \mathbb{Z}_{+}$. We denote by $J^{0}$ this matrix and by $K_{i, j}^{0}$ the corresponding elements $K_{i, j}$. Also denote by $L_{0}^{0}$ the operator $L_{0}$ generated by $J^{0}$. It follows from (17)-(18) that $K_{i, i-2 l}^{0}=0, l=0, \ldots,[i / 2]$. In this case, using (15),(18), we find the formulae for $K_{i, j}^{0}$ in terms of $a_{n}$

$$
K_{i, j}^{0}=(-1)^{\left[\frac{i-j}{2}\right]} \delta_{p+1,2} \frac{a_{i-2} a_{i-4} \ldots a_{j+1}}{a_{i-1} a_{i-3} \ldots a_{j}}, \quad i>j+1, \quad p \equiv i-j(\bmod 2), K_{i, i-1}^{0}=\frac{1}{a_{i-1}} .
$$

If we take the matrix $J$ such that $\left|b_{n}\right|<C<\infty, n \in \mathbb{Z}_{+}$, then one may consider the corresponding operator $L_{0}$ as a perturbation of $L_{0}^{0}$ by a bounded symmetric $\left(b_{n} \in \mathbb{R}\right)$ operator. Then, as known, the deficiency indices of $L_{0}$ are the same as of $L_{0}^{0}$, and applying the Theorem 2, we come to the following conclusion.

Theorem 3. Assume that the diagonal elements of the matrix J are uniformly bounded. Then, the corresponding operator $L_{0}$ is self-adjoint iff there exists a sequence of intervals of positive integers $\left[n_{k}, m_{k}\right]$ such that $m_{k} \leq$ $n_{k+1}<m_{k+1},(k \in \mathbb{N})$ for which the following condition is satisfied:

$$
\sum_{k=1}^{\infty}\left(\sum_{i=n_{k}}^{m_{k}} \sum_{j=n_{k}}^{i}\left|K_{i, j}^{0}\right|^{2}\right)^{1 / 2}=\infty,
$$

where $K_{i, j}^{0}$ are calculated by (21).

Note that the claim of this theorem remains valid for the operators $A=L_{0}^{0}+B$, where $B$ is an arbitrary bounded Hermitian operator in $l^{2}[0, \infty)$ defined on all the space.

\section{Limit-circle case. Self-adjoint extensions.}

Here we consider the non-self-adjoint case for $L_{0}$. As mentioned in the previous section, the deficiency indices of this operator are then equal to $(1,1)$. 
According to the Theorem 1, if the deficiency indices of $L_{0}$ are (1,1), then both $P(\lambda)$ and $Q(\lambda)$ are in $l^{2}[0, \infty)$ for all $\lambda \in \mathbb{C}$. It follows that $P(\lambda)$ and $Q(\lambda)$ are in $D$. Let $f$ be an arbitrary element in $l^{2}[0, \infty)$. Fix $\lambda=\lambda_{0} \in \mathbb{R}$ and denote by $y=\left(y_{n}\right)_{n=0}^{\infty}$ the solution of $J y=\lambda_{0} y+f$, satisfying the condition $y_{0}=0$. One can easily check that

$$
y_{n}=\sum_{j=0}^{n}\left(P_{j}\left(\lambda_{0}\right) Q_{n}\left(\lambda_{0}\right)-P_{n}\left(\lambda_{0}\right) Q_{j}\left(\lambda_{0}\right)\right) f_{j}=\sum_{j=0}^{n} K_{n, j}\left(\lambda_{0}\right) f_{j} ;
$$

and $y \in l^{2}[0, \infty)$. Therefore, $y \in D$. Using (4), we get

$$
\begin{aligned}
\left(P\left(\lambda_{0}\right), f\right) & =\left(P\left(\lambda_{0}\right), J y-\lambda_{0} y\right)=\left(J P\left(\lambda_{0}\right), y\right)+\left[P\left(\lambda_{0}\right), y\right]_{\infty}-\lambda_{0}\left(P\left(\lambda_{0}\right), y\right)= \\
& =\lambda_{0}\left(P\left(\lambda_{0}\right), y\right)+\left[P\left(\lambda_{0}\right), y\right]_{\infty}-\lambda_{0}\left(P\left(\lambda_{0}\right), y\right)=\left[P\left(\lambda_{0}\right), y\right]_{\infty} ; \\
\left(Q\left(\lambda_{0}\right), f\right) & \left.=\left(Q\left(\lambda_{0}\right), J y-\lambda_{0} y\right)=U Q\left(\lambda_{0}\right), y\right)+\left[Q\left(\lambda_{0}\right), y\right]_{\infty}-\lambda_{0}\left(Q\left(\lambda_{0}\right), y\right)= \\
& =\lambda_{0} y_{0}+\left[Q\left(\lambda_{0}\right), y\right]_{\infty}=\left[Q\left(\lambda_{0}\right), y\right]_{\infty} .
\end{aligned}
$$

Since $P\left(\lambda_{0}\right)$ and $Q\left(\lambda_{0}\right)$ are linearly independent, for any $\alpha$ and $\beta \in \mathbb{C}$ there exists a vector $f$ such that $\left(P\left(\lambda_{0}\right), f\right)=\alpha$ and $\left(Q\left(\lambda_{0}\right), f\right)=\beta$. Then, it follows from the above formulae that $\left[P\left(\lambda_{0}\right), y\right]_{\infty}$ and $\left[Q\left(\lambda_{0}\right), y\right]_{\infty}$ can take any values for an arbitrary $y \in D$. It follows from (2) that $\left[P\left(\lambda_{0}\right), Q\left(\lambda_{0}\right)\right]_{N}=1$ for all $N \in \mathbb{Z}_{+}$. Using this fact, it can be verified that for arbitrary $y$ and $z$ from $l^{2}[0, \infty)$

$$
[y, z]_{N}=-\left[y, P\left(\lambda_{0}\right)\right]_{N}\left[Q\left(\lambda_{0}\right), z\right]_{N}+\left[y, Q\left(\lambda_{0}\right)\right]_{N}\left[P\left(\lambda_{0}\right), z\right]_{N} .
$$

Passing to the limit in (22) as $N \rightarrow \infty$, we find that the condition (5) which determines the domain $D_{0}$ is equivalent to

$$
-\left[y, P\left(\lambda_{0}\right)\right]_{\infty}\left[Q\left(\lambda_{0}\right), z\right]_{\infty}+\left[y, Q\left(\lambda_{0}\right)\right]_{\infty}\left[P\left(\lambda_{0}\right), z\right]_{\infty}=0 .
$$

Since the numbers $\left[Q\left(\lambda_{0}\right), z\right]_{\infty}$ and $\left[P\left(\lambda_{0}\right), z\right]_{\infty}$ can be arbitrary, the latter equality is fulfilled if and only if $\left[y, P\left(\lambda_{0}\right)\right]_{\infty}$ and $\left[y, Q\left(\lambda_{0}\right)\right]_{\infty}$ are equal to zero, and we come to the following conclusion.

Theorem 4. If the deficiency indices of $L_{0}$ are $(1,1)$, then its domain $D_{0}$ consists of the elements $y \in D$, which satisfy the conditions:

$$
\left[y, P\left(\lambda_{0}\right)\right]_{\infty}=0, \quad\left[y, Q\left(\lambda_{0}\right)\right]_{\infty}=0
$$

for any $\lambda_{0} \in \mathbb{R}$.

Note that for $\lambda_{0}=0$ this theorem was proved in [5] (Theorem 1.1). Now we recall the definition of a space of boundary values (SBV) of an operator (see e. g. [10], page 155). In particular, this concept is used in study of extensions of the symmetric operators with equal deficiency indices. Namely, let $A$ be a closed symmetric operator on $\mathcal{H}$. Then, a triple $\left(\tilde{\mathcal{H}}, \Gamma_{1}, \Gamma_{2}\right)$, where $\tilde{\mathcal{H}}$ is a Hilbert space and $\Gamma_{1}, \Gamma_{2}$ are linear mappings of $D\left(A^{*}\right)$ into $\tilde{\mathcal{H}}$, is called a boundary value space of the operator $A$, if:

1. for any $f, g \in D\left(A^{\star}\right)$

$$
\left(A^{\star} f, g\right)-\left(f, A^{\star} g\right)=\left(\Gamma_{1} f, \Gamma_{2} g\right)_{\tilde{\mathcal{H}}}-\left(\Gamma_{2} f, \Gamma_{1} g\right)_{\tilde{\mathcal{H}}} ;
$$

2. for any $F_{1}, F_{2} \in \tilde{\mathcal{H}}$ there exists a vector $f \in D\left(A^{\star}\right)$ such that $\Gamma_{1} f=F_{1}, \Gamma_{2} f=F_{2}$.

It is known, that for any operator $A$ with deficiency indices $(n, n), n \leq \infty$ there exists a space of boundary values $\left(\tilde{\mathcal{H}}, \Gamma_{1}, \Gamma_{2}\right)$, such that $\operatorname{dim} \tilde{\mathcal{H}}=n$ (see [5], Chapter 3). Thus, for the operators $L_{0}$ in the limit-circle case, one may take $\tilde{\mathcal{H}}=\mathbb{C}$. It follows from (4) and (22) (in the limit case) that

$$
\begin{aligned}
& (L y, z)-(y, L z)=(J y, z)-(y, J z)= \\
= & {\left[y, P\left(\lambda_{0}\right)\right]_{\infty}\left[Q\left(\lambda_{0}\right), z\right]_{\infty}-\left[y, Q\left(\lambda_{0}\right)\right]_{\infty}\left[P\left(\lambda_{0}\right), z\right]_{\infty}=} \\
= & {\left[y, Q\left(\lambda_{0}\right)\right]_{\infty} \overline{\left[z, P\left(\lambda_{0}\right)\right]_{\infty}}-\left[y, P\left(\lambda_{0}\right)\right]_{\infty} \overline{\left[z, Q\left(\lambda_{0}\right)\right]_{\infty}} }
\end{aligned}
$$

for $y$ and $z$ in $D=D(L)=D\left(L_{0}^{\star}\right)$. Thus, if for $y \in D$ we define $\Gamma_{1} y:=\left[y, Q\left(\lambda_{0}\right)\right], \Gamma_{2} y:=\left[y, P\left(\lambda_{0}\right)\right]$, we find that the triple $\left(\mathbb{C}, \Gamma_{1}, \Gamma_{2}\right)$ is a SBV of $L_{0}$. 
Applying to our case the Theorem 1.6 of [10] (page 156), which describes the extensions of symmetric operators in terms of their SBV, we get the following result.

Theorem 5. If the deficiency indices of the operator $L_{0}$ are (1,1), then for any $\lambda_{0} \in \mathbb{R}$ there exists a self-adjoint extension $L_{0}^{\text {ext }}\left(\lambda_{0}, h\right)$ of $L_{0}$, determined by the equality $L_{0}^{\text {ext }}\left(\lambda_{0}, h\right)=L$ on the elements $y \in D$ satisfying the boundary condition:

$$
\Gamma_{1} y-h \Gamma_{2} y=\left[y, Q\left(\lambda_{0}\right)\right]_{\infty}-h\left[y, P\left(\lambda_{0}\right)\right]_{\infty}=0,
$$

where $h$ is a real number or infinity. In the latter case, the condition (24) takes the form

$$
\left[y, P\left(\lambda_{0}\right)\right]_{\infty}=0 .
$$

Also note that according to the above mentioned theorem, every self-adjoint extension of $L_{0}$ can be described in terms of the Theorem 5 (see [10] for the details).

\section{Commuting Jacobi operators}

We start with two Jacobi matrices:

$$
J^{1}=\left(\begin{array}{cccc}
b_{0}^{1} & a_{0}^{1} & 0 & 0 \\
a_{0}^{1} & b_{1}^{1} & a_{1}^{1} & 0 \\
0 & \ddots & \ddots & \ddots
\end{array}\right), \quad J^{2}=\left(\begin{array}{cccc}
b_{0}^{2} & a_{0}^{2} & 0 & 0 \\
a_{0}^{2} & b_{1}^{2} & a_{1}^{2} & 0 \\
0 & \ddots & \ddots & \ddots
\end{array}\right) .
$$

As in the previous section, denote by $L_{0}^{1}$ and $L_{0}^{2}$ the minimal closed symmetric operators in $l^{2}[0, \infty)$, generated by $J^{1}$ and $J^{2}$ respectively. Also, denote by $P^{1}(\lambda)=\left(P_{i}^{1}(\lambda)\right)_{i=0}^{\infty}, Q^{1}(\lambda)=\left(Q_{i}^{1}(\lambda)\right)_{i=0}^{\infty}$ and $P^{2}(\lambda)=$ $\left(P_{i}^{2}(\lambda)\right)_{i=0}^{\infty}, Q^{2}(\lambda)=\left(Q_{i}^{2}(\lambda)\right)_{i=0}^{\infty}$ the systems of polynomials of the first and the second kind, corresponding to $J^{1}$ and $J^{2}$. The formal matrix commutativity $J^{1} J^{2}=J^{2} J^{1}$ (which amounts to commutativity of $L_{0}^{1}$ and $L_{0}^{2}$ on the set $D_{0}^{\prime}$ ) yields

$$
a_{n}^{2}=k a_{n}^{1}, \quad b_{n}^{2}=k b_{n}^{1}-b ;
$$

where $k=a_{0}^{2} / a_{0}^{1}>0, b=k b_{0}^{1}-b_{0}^{2}$. One can easily check that if the conditions (25) are fulfilled, then $D^{1}=D^{2}$, where $D^{1}=D\left(L^{1}=\left(L_{0}^{1}\right)^{\star}\right), D^{2}=D\left(L^{2}=\left(L_{0}^{2}\right)^{\star}\right)$. Also, for $y, z \in D^{2}$ we have $[y, z]_{\infty}^{2}=k[y, z]_{\infty}^{1}$, where [., . $]_{\infty}^{i}:=[., \text {. }]_{\infty}$ for $L_{0}^{i}, i=1,2$. Thus, we find from (5) that the domains $D_{0}^{1}$ and $D_{0}^{2}$ of $L_{0}^{1}$ and $L_{0}^{2}$ coincide (we denote their domain by $D_{0}^{1,2}$ ). In terms of the above polynomial systems, (25) implies that

$$
P_{i}^{2}(\lambda)=P_{i}^{1}\left(\frac{\lambda}{k}+\lambda_{1}\right) \quad Q_{i}^{2}(\lambda)=\frac{1}{k} Q_{i}^{1}\left(\frac{\lambda}{k}+\lambda_{1}\right) ; \quad \lambda_{1}=\frac{b}{k} ; \quad i \in \mathbb{Z}_{+} .
$$

For the elements of $\operatorname{PFC}\left(J^{1}, \tilde{P}^{1}(\lambda)\right)=\left(\left\{K_{n, n-2}^{1}\right\},\left\{K_{n, n-1}^{1}\right\}\right)_{n=1}^{\infty}$ and $\operatorname{PFC}\left(J^{2}, \tilde{P}^{2}(\lambda)\right)=\left(\left\{K_{n, n-2}^{2}\right\},\left\{K_{n, n-1}^{2}\right\}\right)_{n=1}^{\infty}$ we find, using (15),(17), that

$$
\begin{aligned}
K_{n, n-1}^{2} & =\frac{1}{k} K_{n, n-1}^{1} ; \quad n \in \mathbb{N} ; \\
K_{n, n-2}^{2} & =\frac{1}{k}\left(K_{n, n-2}^{1}+\frac{\lambda_{1}}{a_{n-1}^{1} a_{n-2}^{1}}\right)=\frac{1}{k}\left(K_{n, n-2}^{1}+\lambda_{1} K_{n, n-1}^{1} K_{n, n-2}^{1}\right) .
\end{aligned}
$$

From (26) and the Theorem 1 follows, that the deficiency indices of $L_{0}^{1}$ and $L_{0}^{2}$ are the same, in particular, if $L_{0}^{1}$ is self-adjoint, then so is $L_{0}^{2}$, and vice versa. In the latter case, the commutativity of $L_{0}^{1}$ and $L_{0}^{2}$ follows from Nussbaum's theorem; one should set $\mathcal{D}=D_{0}^{\prime}, \bar{A}=L_{0}^{1}, \bar{B}=L_{0}^{2}$. Then $D(T)=D(A)=D(B)=D_{0}^{1,2}$ and all conditions of the theorem are satisfied. Also note that if $L_{0}^{1}$ and $L_{0}^{2}$ are self-adjoint, then we find from (25) that $L_{0}^{2}=k L_{0}^{1}+b E$, where $E$ is the identity operator, and the commutativity of $L_{0}^{1}$ and $L_{0}^{2}$ can be easily derived from the spectral theorem for self-adjoint operators. 
Now consider the non-self-adjoint case for $L_{0}^{1}$ and $L_{0}^{2}$. We assume that for $J^{1}$ and $J^{2}$ the conditions (25) are satisfied. Further on it will be shown that $L_{0}^{1}$ and $L_{0}^{2}$, admit a commuting self-adjoint extensions. As in the self-adjoint case, we have that $L^{2}=k L^{1}+b E, D^{1}=D^{2}, \quad[., .]_{\infty}^{2}=k[., .]_{\infty}^{1}$ and $D_{0}^{1}=D_{0}^{2}$.

Fix an arbitrary $h \in \mathbb{R}$ and for $\lambda_{0}=0$ consider the extension $L_{0}^{\text {ext,2}}(0, h)$ of $L_{0}^{2}$, defined according to the Theorem 5. It follows from (26) that

$$
\begin{aligned}
{\left[y, Q^{2}(0)\right]_{\infty}^{2}-h\left[y, P^{2}(0)\right]^{2} } & =k\left[y, \frac{1}{k} Q^{1}\left(\lambda_{1}\right)\right]_{\infty}^{1}-h k\left[y, P^{1}\left(\lambda_{1}\right)\right]_{\infty}^{1}= \\
& =\left[y, Q^{1}\left(\lambda_{1}\right)\right]_{\infty}^{1}-h k\left[y, P^{1}\left(\lambda_{1}\right)\right]_{\infty}^{1} .
\end{aligned}
$$

Since $D^{1}=D^{2}$, we conclude from the Theorem 5 that $L_{0}^{\text {ext,1 }}\left(\lambda_{1}, k h\right)$ is a self-adjoint extension of $L_{0}^{1}$ and $D\left(L_{0}^{\text {ext,2}}(0, h)\right)=D\left(L_{0}^{\text {ext, }}\left(\lambda_{1}, k h\right)\right)$. Applying the same arguments as in the case of self-adjoint $L_{0}^{1}$ and $L_{0}^{2}$, we find that $L_{0}^{\text {ext, } 1}\left(\lambda_{1}, k h\right)$ and $L_{0}^{\text {ext, }}(0, h)$ are commute. For $h=\infty$, the commutativity of $L_{0}^{\text {ext, } 1}\left(\lambda_{1}, \infty\right)$ and $L_{0}^{\text {ext }, 2}(0, \infty)$ follows from the above arguments and the relation

$$
\left[y, P^{2}(0)\right]_{\infty}^{2}=k\left[y, P^{1}\left(\lambda_{1}\right)\right]_{\infty}^{1} .
$$

In view of the above, we can conclude that $L_{0}^{1}$ and $L_{0}^{2}$ admit a commuting self-adjoint extensions. Thus we obtain the main result of this section.

Theorem 6. Assume that for $J^{1}$ and $J^{2}$ the conditions (25) are fulfilled. Then, if the deficiency indices of $L_{0}^{1}$ and $L_{0}^{2}(0,0)$, then they are self-adjoint and commute. If their deficiency indices are (1,1), then for every $h \in \mathbb{R} \cup \infty$ these operators admit a commuting self-adjoint extensions $L_{0}^{\text {ext }, 1}\left(\lambda_{1}, k h\right)$ and $L_{0}^{\text {ext }, 2}(0, h)$, defined according to the Theorem 5 , and $\lambda_{1}=b_{0}^{1}-b_{0}^{2} / k \in \mathbb{R}$.

As to the theorem of Nussbaum, in the limit-circle case it cannot be applied directly since the operator $T$, defined as the sum of $L_{0}^{1}$ and $L_{0}^{2}$, is not self-adjoint. However, the proof of Nussbaum's theorem relies on the following proposition from the same paper [3]:

Proposition, Nussbaum. Let $A$ be a densely defined symmetric operator in a Hilbert space. If $T$ is a self-adjoint operator such that $D(T) \subset D(\bar{A})$ and

$$
\left(T x, A^{\star} y\right)=(\bar{A} x, T y), \quad \forall x, y \in D(T),
$$

then $\bar{A}$ is self-adjoint and commutes with $T$.

In our case, we can set $T=L_{0}^{e x t, 2}(0, h), \bar{A}=L_{0}^{e x t, 1}\left(\lambda_{1}, k h\right)$. Then $D(T)=D(\bar{A})$ and using (22)-(23), we get

$$
\begin{array}{r}
\left(T x, A^{\star} y\right)-(\bar{A} x, T y)=b\left(\left(x, J^{1} y\right)-\left(J^{1} x, y\right)\right)=b[x, y]_{\infty}^{1}= \\
=\frac{b}{k}\left(\left[x, Q^{2}\left(\lambda_{0}\right)\right]_{\infty}^{2}\left[P^{2}\left(\lambda_{0}\right), y\right]_{\infty}^{2}-\left[x, P^{2}\left(\lambda_{0}\right)\right]_{\infty}^{2}\left[Q^{2}\left(\lambda_{0}\right), y\right]_{\infty}^{2}\right)= \\
=\frac{b}{k}\left[x, P^{2}\left(\lambda_{0}\right)\right]^{2}\left(h\left[P^{2}\left(\lambda_{0}\right), y\right]_{\infty}^{2}-\left[Q^{2}\left(\lambda_{0}\right), y\right]_{\infty}^{2}\right)=0 .
\end{array}
$$

Thus, all conditions of the above proposition are fulfilled, so it is applicable for our case.

\section{References}

[1] E. Nelson, Analytic vectors, Ann. of Math., 70(3), (1959), 572-615.

[2] M. Reed, B. Simon, Methods of Modern Mathematical Physics, Vol. 1: Functional Analysis, Academic Press, New York (1980).

[3] A.E. Nussbaum, A commutativity theorem for unbounded operators in Hilbert space, Trans. Amer. Math. Soc.,vol. 140, (1969) 485-491. 
[4] Y. Xu, Unbounded commuting operators and multivariate orthogonal polynomials, Proc. Amer. Math Soc., vol. 119, No 4, (1993) 1223-1231.

[5] B.P. Allakhverdiev, G. Sh. Guseinov, On the spectral theory of dissipative difference operators of second order, Math. USSR Sbornik, vol 66, No. 1, (1990), 107-125.

[6] N.I. Akhiezer, The Classical Moment Problem, Oliver Boyd, Edinburgh (1965).

[7] J. Dombrovski, Cyclic operators, commutators, and absolutely continuous measures, Proc. Amer. Math Soc., vol. 100, No 3 , (1987), 457-463.

[8] M. Gekhtman, A. Kalyuzhny, On the orthogonal polynomials in several variables, Integr. Equat. Oper. Theory, vol. 19, Isssue 4, (1994), 404-418.

[9] C.F. Dunkl, Y. Xu, Orthogonal Polynomials of Several Variables, Encyclopedia of Mathematics and its Applications 81, Cambridge University Press, Cambridge (2001).

[10] V.I. Gorbachuk, M.L. Gorbachuk, Boundary Value Problems for Operator Differential Equations, Kluwer Academic Publishers, Dordrecht (1991).

[11] E.N. Petropoulou, L. Velazquez, Self-adjointness of unbounded tridiagonal operators and spectra of their finite truncations, J. Math. Anal. Appl., vol. 420, (2014), 852-872.

[12] Ju. M. Berezanskij, Expansions in Egenfunctions of Selfadjoint Operators, AMS, Providence, R.I. (1968).

[13] E. M. Nikishin, V. N. Sorokin, Rational Approximations and Orthogonality, Translations of Mathematical Monographs, vol. 92, AMS, Providence, R.I. (1991).

[14] A.G. Kostyuchenko, K.F. Mirzoev, Three-term recurrence relations with matrix coefficients. The completely indeterminate case, Math. Notes, vol. 63, No. 5, (1998) 624-630.

[15] A. G. Kostyuchenko, K. A. Mirzoev, Complete indefiniteness tests for Jacobi matrices with matrix entries, Functional Analysis and Its Applications, vol.35, No. 4, (2001) 265-269.

[16] A. Osipov, On the completely indeterminate case for block Jacobi matrices. Concrete Operators, vol. 4, No. 1, (2017) 48-57.

[17] B. Simon, The classical moment problem as a self-adjoint finite difference operator, Adv. Math. 137, (1998) 82-203. 